\title{
Making compact and innovative dual- band thermal imagers using hybrid optical elements
}

Victor Laborde, Jérôme Loicq, Serge Habraken, Gaëtan Kershen

Victor Laborde, Jérôme Loicq, Serge Habraken, Gaëtan Kershen, "Making compact and innovative dual-band thermal imagers using hybrid optical elements," Proc. SPIE 11852, International Conference on Space Optics ICSO 2020, 118522E (11 June 2021); doi: 10.1117/12.2599377

SPIE Event: International Conference on Space Optics - ICSO 2021, 2021, Online Only 


\section{International Conference on Space Optics-ICSO 2020}

Virtual Conference

30 March-2 April 2021

Edited by Bruno Cugny, Zoran Sodnik, and Nikos Karafolas
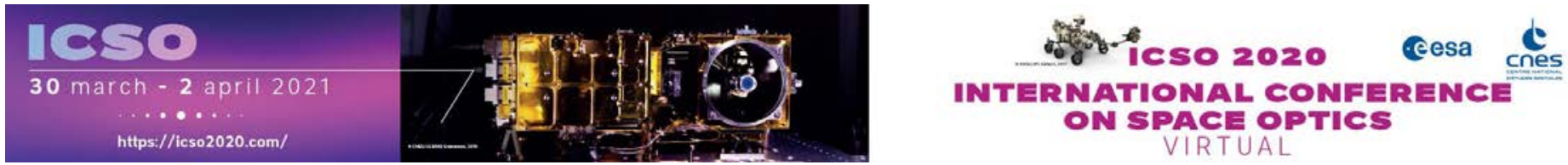

\section{Making compact and innovative dual-band thermal imagers using hybrid optical elements}

\section{Cesa isoporecestings denes}




\title{
Making compact and innovative dual-band thermal imagers using hybrid optical elements
}

\author{
Victor Laborde ${ }^{\mathrm{a}}$, Jérôme Loicq ${ }^{\mathrm{a}}$, Serge Habraken ${ }^{\mathrm{a}}$, and Gaëtan Kershen ${ }^{\mathrm{b}}$ \\ ${ }^{a}$ Centre Spatial de Liege, Avenue du Pré-Aily, 4031 Angleur, Belgium \\ b2Aerospatial and Mechanics department, Faculty of applied Sciences, University of Liege, \\ Belgium
}

\begin{abstract}
Infrared (IR) remote sensing offers a huge range of applications, mostly addressing make-or-break issues of our century (crops water content monitoring, forest fires and volcanic eruption detection and imaging, etc.). These applications fall under different spectral bands, known as mid and long-wave infrared, which are very hard to combine in a single compact instrument. In this article we propose to explore the infrared (IR) behaviour of a dual-band diffractive component: the multilayer diffractive optical element (MLDOE). We use and discuss the thin element approximation as a valid phase model. Using Fourier optics, we are able to simulate the resulting image of the MLDOE. Thereby, ray-tracing software are not accurate to model a complex diffractive component. The Strehl ratio is used to determine the focalization efficiency for the working order, which is above $95 \%$ in the mid and long-wave infrared bands. This result, along with the very low energy content of the other orders, proves the strong imaging potential of MLDOEs for dual-band applications. It is also demonstrated that the MLDOE has the same chromatic behaviour as standard DOEs, making it a very useful component for infrared achromatization.
\end{abstract}

Keywords: Infrared, broadband, imaging, diffractive optics, Fourier optics

\section{INTRODUCTION}

Thermal infrared (TIR) has a wide domain of applications for Earth observation, including soil and atmosphere monitoring, risk assessment and security. For instance mid-wave infrared (MWIR) is the optimal band to image forest fires. ${ }^{1}$ Volcanic eruptions are preceded and followed by geophysical manifestations, which make them mostly detectable in TIR. ${ }^{2}$ Other TIR applications, such as air pollution and decease prevention have been reviewed in. ${ }^{3,4}$ Multispectral spaceborn instruments have already been developed for large space missions such as ENVISAT (AASTR), ${ }^{5}$ AQUA (MODIS), ${ }^{6}$ ADEOS II (GLI) ${ }^{7}$ or SENTINEL 8. These instruments classically have high optical performance with a limited revisit time. In this paper, the two main targeted applications are: the monitoring of crops water content and the imaging of hot events (forest fires, lava flows, etc.). Since $70 \%$ of Earth freshwater is used for irrigation, crop water monitoring is a key humanitarian issue. ${ }^{8,9}$ The second application falls into the security domain and is also crucial. These two applications involve temperature sensing around $300^{\circ} \mathrm{K}$ for crop monitoring and $600^{\circ} \mathrm{K}$ for hot events detection. These temperatures respectively have a peak of emission in the long-wave infrared band (LWIR: 8-12 $\mu \mathrm{m}$ ) and in the mid-wave infrared band (MWIR: 4.4-5 $\mathrm{\mu m}$ ). These two applications also require a daily revisit time, which is only achievable by using a constellation of satellites. In summary, this article is dedicated to the study of an IR dual-band camera, as compact as possible. The main issue of such camera is the compensation of two major defocus: the chromatic focal shift and the thermal focal shift. The most optimal solution is to use diffractive lenses (DOEs), which are compact and able to compensate for the chromatic and thermal defocus of refractive lenses. However, these DOEs do not operate well when the waveband is large. ${ }^{10,11}$ This article aims to describe the model and the image quality of a recent element: the multilayer diffractive optical element (MLDOE). It consists of a broadband

Further author information: (Send correspondence to V. Laborde)

V. Laborde.: E-mail: victor.laborde@uliege.be, Telephone: +32 043824685 
extension of a diffractive lens and was already described in many papers. ${ }^{12-16}$ This paper major results are the expression of the chromatic focal shift of MLDOEs and the study of their image quality.

In Section 2, we describe how to extend the geometric model of kinoform DOEs to the case of a combination of two harmonic DOEs (HDOEs). HDOEs are able to use a higher diffractive order by increasing their profile height. ${ }^{17,18}$ The phase retardation of the MLDOE is expressed under the thin element approximation (TEA), resulting in a scalar paraxial field. In Sections 3 and 4, the validity of the TEA and the Fourier optics propagation algorithm are presented. The scalar field leaving the MLDOE is propagated to the focal plane using the angular spectrum method. ${ }^{19}$ The focalization efficiency is measured through the Strehl ratio and is above 0.96 for both MWIR and LWIR, inside the validity domain of the scalar theory. The study of the chromatic focal shift of MLDOEs is also a major result of this paper and is investigated in Section 5. Finally, the EAM provides an accurate diffraction efficiency expression and is presented in Section 6.

\section{BROADBAND DIFFRACTIVE OPTICAL ELEMENT}

In this Section, we explain the modeling of a diffractive component with multiple layers called a multilayer diffractive optical element (MLDOE). The following reasoning is based on the work of V. Moreno et al. ${ }^{11}$ The MLDOE consists of two harmonic diffractive lens assembled together. They have the same number of Fresnel zones and are made of different materials. The thin element approximation (TEA) is used to compute the optical path difference inside a given Fresnel zone. The main design objective is to force the interferences to be constructive for two distinct "design" wavelengths, chosen inside the waveband. This condition will drive the shape and height of the MLDOE micro-structures, specific to the material combination used.

The geometric model of MLDOE is shown in Figure 1. In this paper we consider any material combination, though in practice a manufacturability issue may arise.

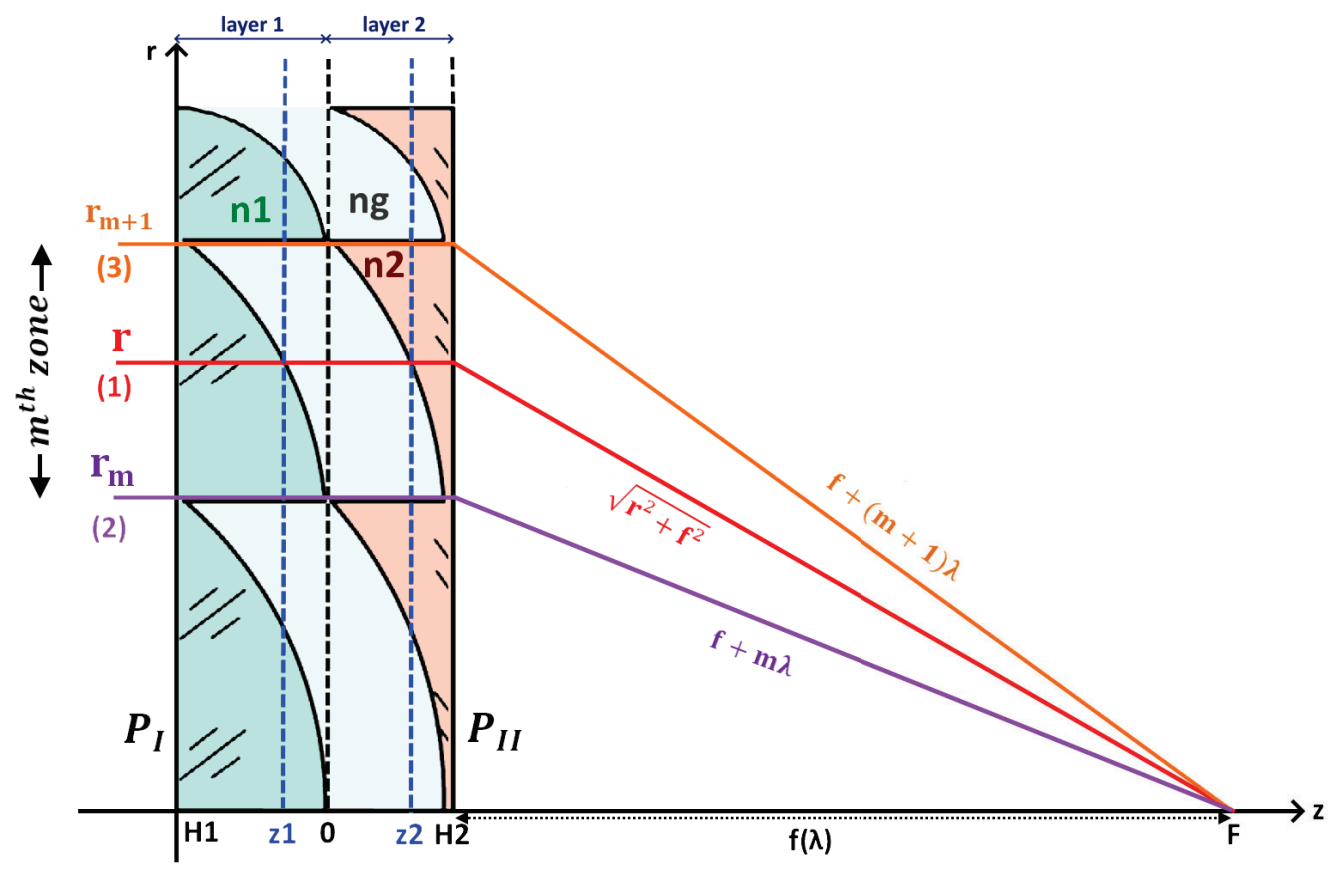

Figure 1: Extended model of DOE to multilayer DOE. The thin element approximation (TEA) implies a paraxial incident beam. The shape of layer $i=1,2$ along the optical axis $z$ at a radius $r$ is described by $z_{i}(r)$. $H_{i}$ is the maximal height of the profile. $f(\lambda)$ is the focal distance of the MLDOE at the wavelength $\lambda$ and $m$ is the considered diffractive zone, starting at a radius $r_{m}$ and finishing at $r_{m+1}$. Each layer $\mathrm{i}$ is made of a distinct material with refractive index $n_{i}(\lambda)$ and the gap is filled with a material $n_{g}(\lambda)$. Interferences between each zone are constructive at the focal plane. 
The TEA states that the element is sufficiently thin so that a paraxial beam propagates without any deviation from $P_{I}$ to $P_{I I}$. For a given wavelength $\lambda$, the interferences at the focal plane are constructive if the optical path difference (OPD) between two consecutive zones is equal to $\lambda$. This recursive zone design gives the radius of the $m^{\text {th }}$ zone:

$$
r_{m}^{2}=2 m f \lambda_{d}+\left(m \lambda_{d}\right)^{2} \simeq 2 m f \lambda_{d}
$$

Where $\mathrm{f}$ is the focal length of the MLDOE defined at the wavelength $\lambda_{d}$, the "design wavelength". The origin is taken between both layers, therefore the optical path length (OPL) is counted negatively inside the first layer and positively inside the second one. The general OPL followed by a ray incident at a radius $r \in\left[r_{m}, r_{m+1}[\right.$ inside the MLDOE is represented by the ray (1) and can be expressed as:

$$
O P L(r)=-\left(n_{1}\left(H_{1}-z_{1}(r)\right)+z_{1}(r) n_{g}\right)+z_{2}(r) n_{g}+n_{2}\left(H_{2}-z_{2}(r)\right)+\sqrt{f^{2}+r^{2}}
$$

$z_{i}, H_{i}, n_{i}$ are respectively the profile height at radius $\mathrm{r}$, the maximal profile height and the refractive index of layer $i$, as shown in Figure 1. $n_{g}$ is the refractive index of the material filling the gap between each layer. Using the ray at the start of the $m^{t h}$ zone (ray (2)) as an arbitrary reference, the optical path difference (OPD) is:

$$
O P D(r)=O P L(r)-O P L\left(r=r_{m}\right)
$$

The expression of the optical path length of any ray incident at a radius $r$ inside the $m^{t h}$ zone is:

$$
\left.O P L(r)=n_{1}\left(H_{1}-z_{1}(r)\right)+n_{g}\left(z_{1}(r)+z_{2}(r)\right)+n_{2}\left(H_{2}-z_{2}\right)+\sqrt{(} f_{d}^{2}+r^{2}\right)
$$

Where $r \in\left[r_{m} ; r_{m+1}[\right.$. Then the optical path difference can be expressed by:

$$
O P D(r)=O P L(r)-O P L\left(r=r_{m}\right)=O P L(r)-\left(n_{1} H_{1}+n_{g} H_{2}+f_{d}+m \lambda_{d}\right)
$$

Where the reference ray has been arbitrarily taken at the start of the $m^{\text {th }}$ zone (ray (2)). It gives for the OPD:

$$
\left.O P D(r)=\left(n_{g}-n_{1}\right) z_{1}+\left(n_{2}-n_{g}\right)\left(H_{2}-z_{2}\right)+\sqrt{(} f_{d}^{2}+r^{2}\right)-f_{d}-m \lambda_{d}
$$

The term inside the square root can be approximated by its second limited expansion term:

$$
\left.\sqrt{(} f_{d}^{2}+r^{2}\right)=f_{d}\left(1+\frac{r^{2}}{2 f_{d}^{2}}\right)=f_{d}+\frac{r^{2}}{2 f_{d}}
$$

Recall that the first zone radius $r_{1}$ was approximated in Equation $1\left(r_{1}=2 f \lambda_{d}\right)$, so we finally have:

$$
O P D(r)=\left(n_{2}-n_{g}\right)\left(H_{2}-z_{2}(r)\right)+z_{1}(r)\left(n_{1}-n_{g}\right)-\lambda_{d}\left(m-\frac{r^{2}}{r_{1}^{2}}\right)
$$

A particular case arises in Equation 7 when $r=r_{m+1}\left(\right.$ ray $(3)$ in Figure 1): $z_{1}\left(r_{m+1}\right)=H_{1}$ and $z_{2}\left(r_{m+1}\right)=0$, leaving:

$$
O P D\left(r_{m+1}\right)=\left(n_{1}-n_{g}\right) H 1+\left(n_{2}-n_{g}\right) H_{2}+\lambda_{d}
$$

The specific shape $z_{1}, z_{2}$ for each layer is a consequence of Fermat's principle. Applied to the $m^{\text {th }}$ zone, it gives:

$$
\forall r \in\left[r_{m}, r_{m+1}\right], O P D(r)=0
$$


Equations 7, 8 and 9 form a system of 4 unknowns and 2 Equations. To obtain a unique shape for each layer, one more constraint must be added. The objective of the research is to create a broad-band device, so the methodology is to use two wavelengths instead of one in the classical kinoform design. Considering that system 9 must be solved for 2 distinct design wavelengths produces two more Equations. $\lambda_{1}$ and $\lambda_{2}$, respectively associated with the focal lengths $f_{1}$ and $f_{2}$ which define these two design wavelengths. Equations 7 and 8 are solved with a unique solution:

$$
\left\{\begin{array}{l}
H_{1}=\left(-\lambda_{1}\left(n_{22}-n_{g 2}\right)+\lambda_{2}\left(n_{21}-n_{g 1}\right)\right) / a \\
H_{2}=\left(+\lambda_{1}\left(n_{12}-n_{g 2}\right)-\lambda_{2}\left(n_{11}-n_{g 1}\right)\right) / a \\
Z_{1}=-H_{1}\left(m-\frac{r^{2}}{r_{1}^{2}}\right) \\
Z_{2}=+H_{2}\left(m-\frac{r^{2}}{r_{1}^{2}}\right)+H_{2}
\end{array}\right.
$$

Where $a$ is defined by: $a=\left(n_{11}-n_{g 1}\right)\left(n_{22}-n_{g 2}\right)-\left(n_{12}-n_{g 2}\right)\left(n_{21}-n_{g 1}\right) \cdot n_{i j}$ describes the index of layer $i$ with respect to wavelength $\lambda_{j}(i, j=1,2)$ and $n_{g j}$ is the filling material index at wavelength $\lambda_{j}$. Finally, $r_{1}$ is defined using relation 1: $r_{1}^{2}=2 f_{1} \lambda_{1}=2 f_{2} \lambda_{2}$.

For any wavelength $\lambda$, the phase delay generated by the MLDOE is expressed as:

$$
\begin{aligned}
\Phi(r, \lambda) & =\frac{2 \pi}{\lambda} O P D(r, \lambda) \\
& =\frac{2 \pi}{\lambda}\left(z_{1}\left(n_{1}-n_{g}\right)+\left(H_{2}-z_{2}\right)\left(n_{2}-n_{g}\right)\right) \\
& =\frac{2 \pi}{\lambda}\left(-H_{1}\left(n_{1}-n_{g}\right)-H_{2}\left(n_{2}-n_{g}\right)\right)\left(m-\frac{r^{2}}{r_{1}^{2}}\right)
\end{aligned}
$$

from this expression, it clearly appears that the MLDOE is an extension of the diffractive lens. Finally, the scalar diffraction efficiency for the $j^{\text {th }}$ diffractive order is given by:

$$
\eta_{j}\left(\lambda, \lambda_{1}, \lambda_{2}, j\right)=\operatorname{sinc}^{2}\left(\frac{-H_{1}\left(n_{1}-n_{g}\right)-H_{2}\left(n_{2}-n_{g}\right)}{\lambda}-j\right)
$$

Based on the expression of $H_{1}, H_{2}$ and the parameter $a$ defined in equation 10, the developed expression of diffraction efficiency for a given wavelength $\lambda$ is:

$$
\left\{\begin{aligned}
\eta_{j}(\lambda, j) & =\operatorname{sinc}^{2}\left(\frac{\lambda_{1}}{\lambda a} T_{1}(\lambda)-\frac{\lambda_{2}}{\lambda a} T_{2}(\lambda)-j\right) \\
T_{1}(\lambda) & =\left(n_{22}-n_{g 2}\right)\left(n_{1 \lambda}-n_{g \lambda}\right)-\left(n_{12}-n_{g 2}\right)\left(n_{2 \lambda}-n_{g \lambda}\right) \\
T_{2}(\lambda) & =-\left(n_{21}-n_{g 1}\right)\left(n_{1 \lambda}+n_{g \lambda}\right)-\left(n_{11}-n_{g 1}\right)\left(n_{2 \lambda}-n_{g \lambda}\right)
\end{aligned}\right.
$$

$T_{1}$ and $T_{2}$ depends only on the refractive indices and $a$ is the parameter defined in equation 10 . This expression has a simplified form in the particular cases $\lambda=\lambda_{1}, \lambda_{2}$ :

- $T_{1}\left(\lambda_{2}\right)=0$ and $T_{1}\left(\lambda_{1}\right)=a$.

- $T_{2}\left(\lambda_{1}\right)=0$ and $T_{2}\left(\lambda_{2}\right)=-a$.

It gives the expression of the diffraction efficiency of the MLDOE when the operating wavelengths are considered: 


$$
\left\{\begin{array}{l}
\eta_{j}\left(\lambda_{1}, j\right)=\operatorname{sinc}^{2}\left(\frac{1}{\lambda_{1}}\left[\frac{\lambda_{1}}{a} * a-\frac{\lambda_{2}}{a} * 0\right]-j\right)=\operatorname{sinc}^{2}(1-j) \\
\eta_{j}\left(\lambda_{2}, j\right)=\operatorname{sinc}^{2}\left(\frac{1}{\lambda_{2}}\left[\frac{\lambda_{1}}{a} * 0-\frac{\lambda_{2}}{a} *(-a)\right]-j\right)=\operatorname{sinc}^{2}(1-j)
\end{array}\right.
$$

Therefore, the theoretical diffraction efficiency reaches $100 \%$ for the order $j=1$ and $0 \%$ for any other order when the operating wavelengths are used. So far we have demonstrated a method to design an MLDOE with optimal efficiency given two distinct materials and two design wavelengths. The full plot of the diffraction efficiency for different orders and wavelengths is shown in Figure 2 and is based on Equation 12 . The analysed MLDOE is composed of one zinc selenide $(\mathrm{ZnS})$ layer and one germanium (Ge) layer, separated by air. The design wavelengths have been chosen at $4.7 \mu \mathrm{m}$ and $10.4 \mu \mathrm{m}$. These two design wavelengths are kept for the simulations.

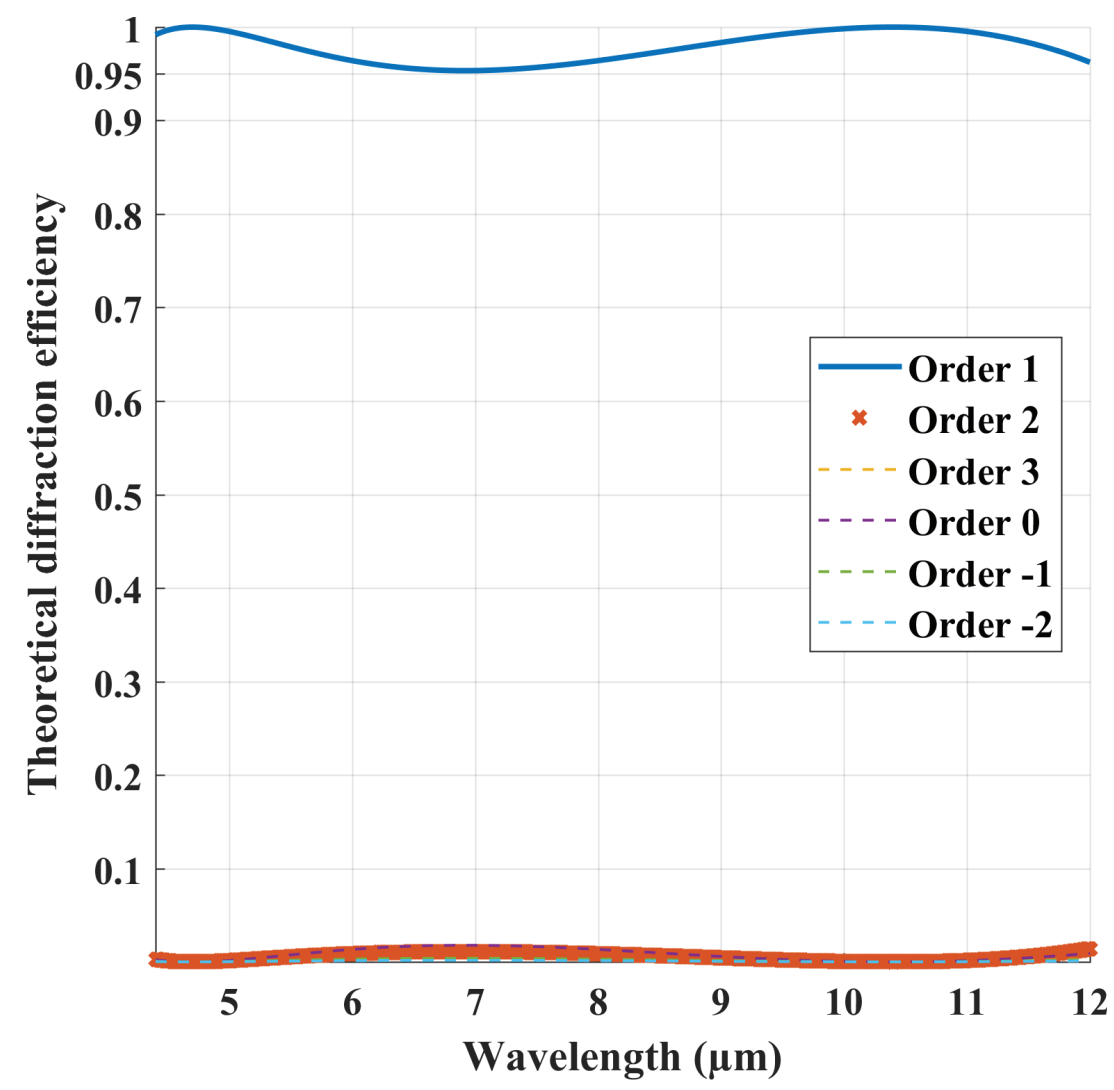

Figure 2: Diffraction efficiency of a ZnS-Air-Ge MLDOE designed in MWIR and LWIR wavebands. The design wavelength are at $4.7 \mu \mathrm{m}$ and $10.4 \mu \mathrm{m}$. The orders refer to the global MLDOE diffractive order. The diffraction efficiency is maximal for both design wavelength and stays above $95 \%$ in the considered infrared band.

Figure 2 represents the variation of the diffraction efficiency with the wavelength. Only the first order operates which is a strong requirement for an imaging optical component. Hence it is demonstrated that the MLDOE is an imaging diffractive element that could theoretically reach above $95 \%$ efficiency in the whole thermal infrared bandwidth. Using Equation 10, the algebraic microstructure heights are $H_{1}=-121 \mu \mathrm{m}$ and $H_{2}=51.8 \mu \mathrm{m}$, respectively for the $\mathrm{ZnS}$ and the Ge layer.

\section{PHASE PROPAGATION METHOD AND VALIDITY OF THE MODEL}

Ray tracing software (CODE V, ZEMAX) do not accurately model the diffraction efficiency of complex diffractive elements. This article proposes a diffraction-based analysis of MLDOEs using Fourier optics. ${ }^{19}$ The treatment 
of monochromatic scalar fields is represented in Figure 3:

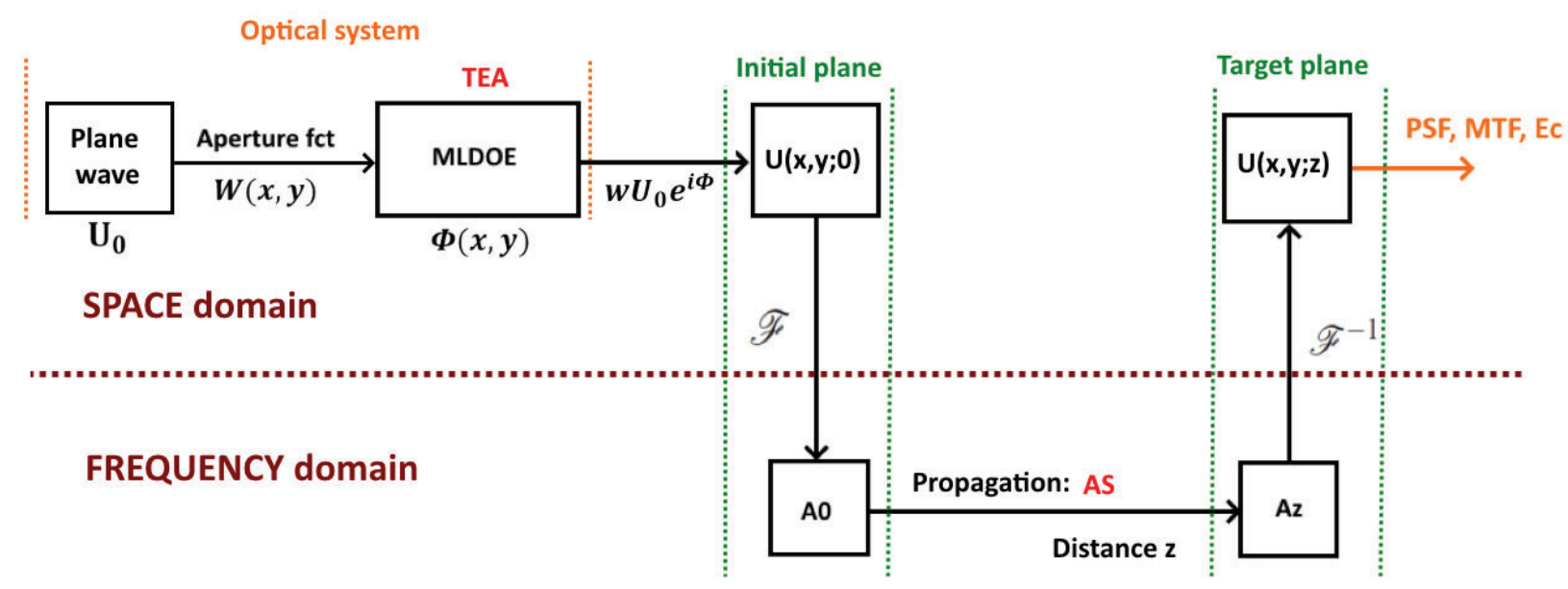

Figure 3: Diagram showing the treatment of a monochromatic scalar field by Fourier optics. The multilayer diffractive optical element (MLDOE) is represented in thin element approximation (TEA) by a phase function $\Phi(x, y)$ (Equation 11) inside a circular aperture $W(x, y)$. The resulting paraxial field in the input plane is propagated in the frequency domain using the angular spectrum (AS) method ${ }^{19}$ over a distance $\mathrm{z}$ along the optical axis. The field $U(x, y ; z)$ is evaluated in the target plane using different metrics (point spread function (PSF), modulation transfer function (MTF), encircled energy,...).

The angular spectrum method accuracy depends on both the precision of the numerical sampling and the physical validity of the input phase delay to be propagated.

The angular spectrum (AS) method requires a fine sampling and a computation window larger than the aperture size to avoid numerical errors caused by the edges. These aspects are not treated in this paper, but the recommendations of Matsushima et all. ${ }^{20}$ have been applied.

In our case, the phase of the MLDOE was modelled according to the thin element approximation. However, the height of each diffractive layer is very high $(100 \mu \mathrm{m})$ compared to the case of a single diffractive lens ( 10 $\mu \mathrm{m})$. In that scenario, the validity of the TEA is questionable, especially when the zone period size gets low. According to ${ }^{21}$ the TEA model is valid when three restrictions are met :

- The microstructure periods are much larger than the wavelength (more than 14 times $^{22}$ )

- The field is paraxial

- The microstructures are thin: their periods must be at least 6 time larger than their heights $\left({ }^{23}\right)$

These restrictions limit the possible design values for the chosen MLDOE, regarding the number of zones and aperture diameter. For instance, with a $14 \mathrm{~mm}$ diameter MLDOE, the TEA restrictions impose a maximum of 5 zones. The period size variation is depicted in Figure 4. The continuous and dashed lines display a threshold corresponding respectively to the first and last restriction.

This design involves a very high $f$-number: $\mathrm{F} / 43.8$. A higher number of zones would decrease the MLDOE $f$-number but the period size would drop below the dashed TEA-limit line.

Finally, the simulation uses $M=1024$ uniformly distributed samples in each direction. The MLDOE pupil diameter and the sampling window size are respectively $D=14 \mathrm{~mm}$ and $S=21 \mathrm{~mm}$. The number of zones is 5 ,

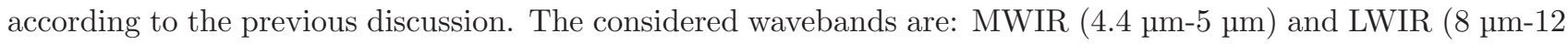
$\mu \mathrm{m})$. 


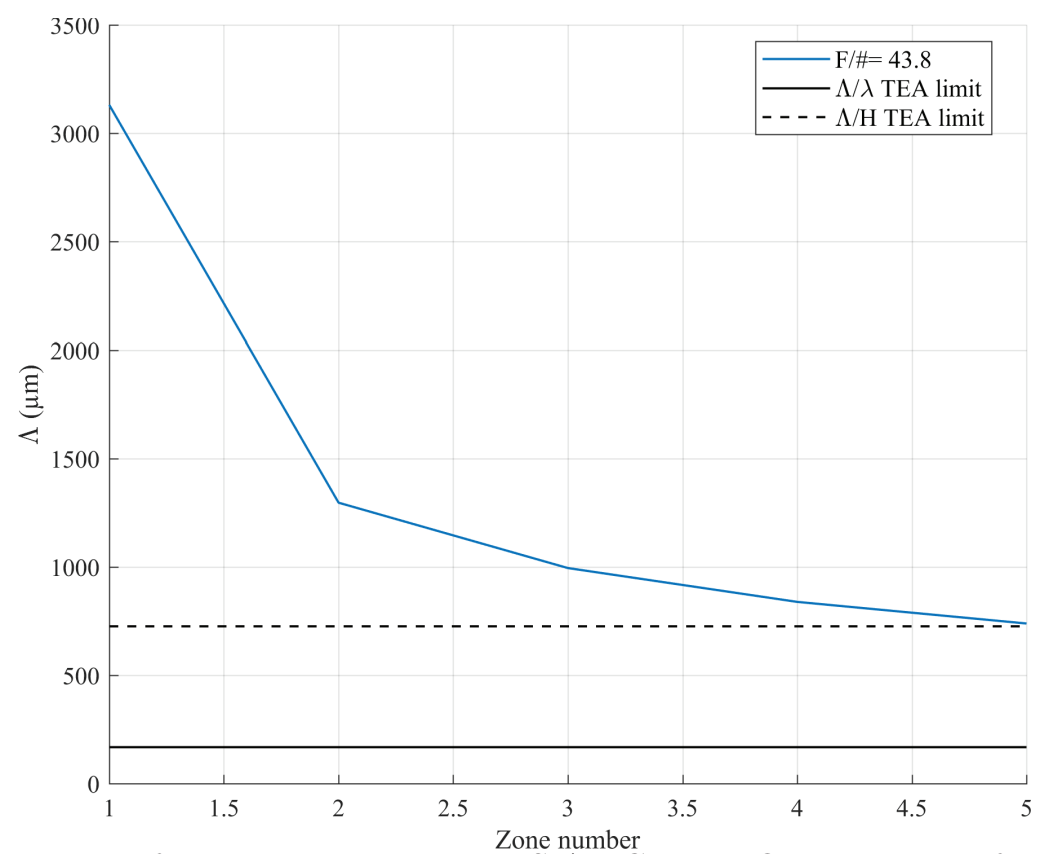

Figure 4: Period size variation for a $14 \mathrm{~mm}$ diameter ZnS-Air-Ge MLDOE composed of 5 diffractive zones. The straight lines display the period threshold above which the scalar theory remains valid considering: the period to wavelength ratio (continuous line) and the period to height ratio (dashed line).

\section{SIMULATION RESULTS}

As previously mentioned, the ZnS-Air-Ge MLDOE is designed at $\lambda_{1}=4.7 \mu \mathrm{m}$ for MWIR and $\lambda_{2}=10.4 \mu \mathrm{m}$ for LWIR. The total energy content of the field in the initial plane is normalized to $1 \mathrm{~W}$ and the aperture is circular. The angular spectrum method is conservative, therefore the total energy of the field at any propagation plane remains the same.

The phase (modulo- $2 \pi$ ) of the field in the initial plane $z=0 \mathrm{~mm}$ is shown in Figure 5 :

The phase delay applied by the MLDOE is null outside of the pupil and follows the kinoform profile (Equation 11) inside.

\subsection{Image at the target plane}

Using the simulation parameters chosen at the end of Section 3, the focal length of the MLDOE at $8 \mu \mathrm{m}$ is $f=620 \mathrm{~mm}$. The irradiance at $8 \mu \mathrm{m}$ and the Strehl ratio for the whole bandwidth are displayed in Figure 6 :

The PSF displays a Bessel pattern, caused by the diffraction effect of the circular aperture. The Strehl ratio for the MLDOE remains above 0.96 for the whole waveband, with maximal values located at the design wavelengths. The Strehl ratio measures the focalization efficiency and leaves only the intrinsic MLDOE diffraction efficiency, hiding the aperture diffraction effect. The MLDOE approaches the diffraction limit, meaning that it has very good imaging capabilities inside the validity domain of the TEA.

\subsection{Results along the optical axis}

In this Section, Fourier optics is used to model the light behaviour along the optical axis. In particular, the study concentrates on the energy contained in the unwanted diffractive orders. The same input field at $8 \mu \mathrm{m}$ is propagated from plane to plane along the optical axis, with $P=620$ planes between the input plane $z=0 \mathrm{~mm}$ and the image plane $z=620 \mathrm{~mm}$ (focal distance). The propagation step $d z=1 \mathrm{~mm}$ is small enough to obtain a "continuous" representation of the field along the optical axis. For each plane, the 2 D irradiance $|U|^{2}$ is recorded in a central slice $y=M / 2+1$, as well as the Strehl ratio. Figure 7 shows a) the logarithm of the irradiance pattern and b) the Strehl ratio along the optical axis: 


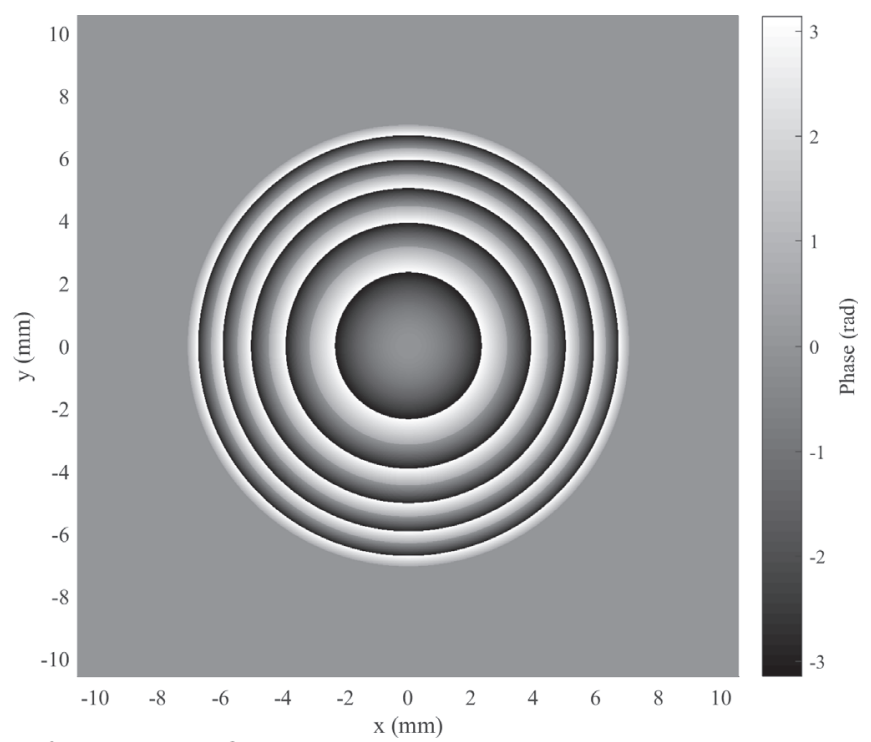

Figure 5: Phase modulo- $2 \pi$ of the input field in the initial plane $z=0 \mathrm{~mm}, \lambda=8 \mu \mathrm{m}$. The phase represents the total phase shift caused by the MLDOE on a paraxial incident plane wave. The phase is calculated using Section 2 Equation 11 under the TEA.

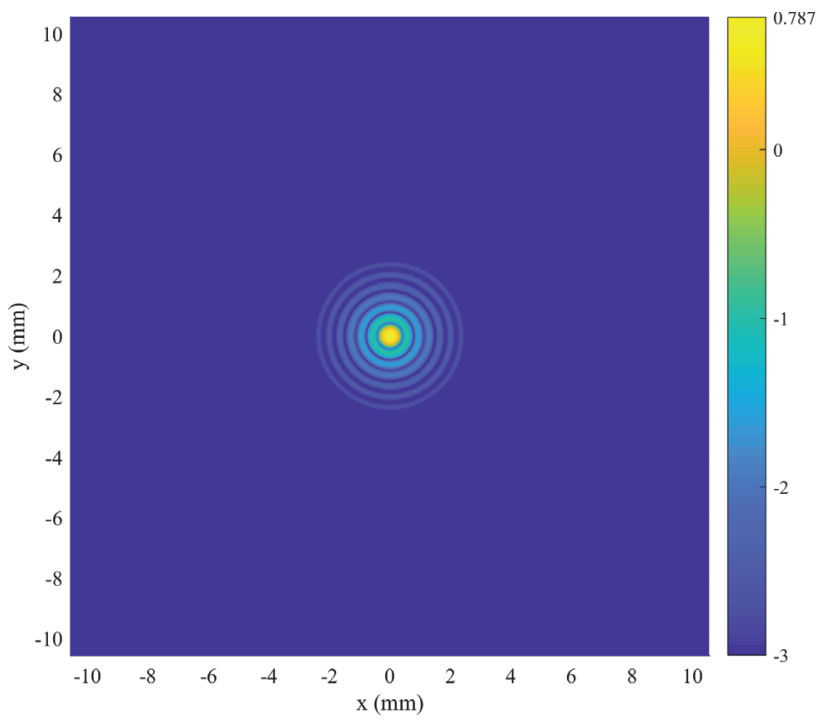

(a) Irradiance $|U(x, y ; z)|^{2}$ at $z=620 \mathrm{~mm}$

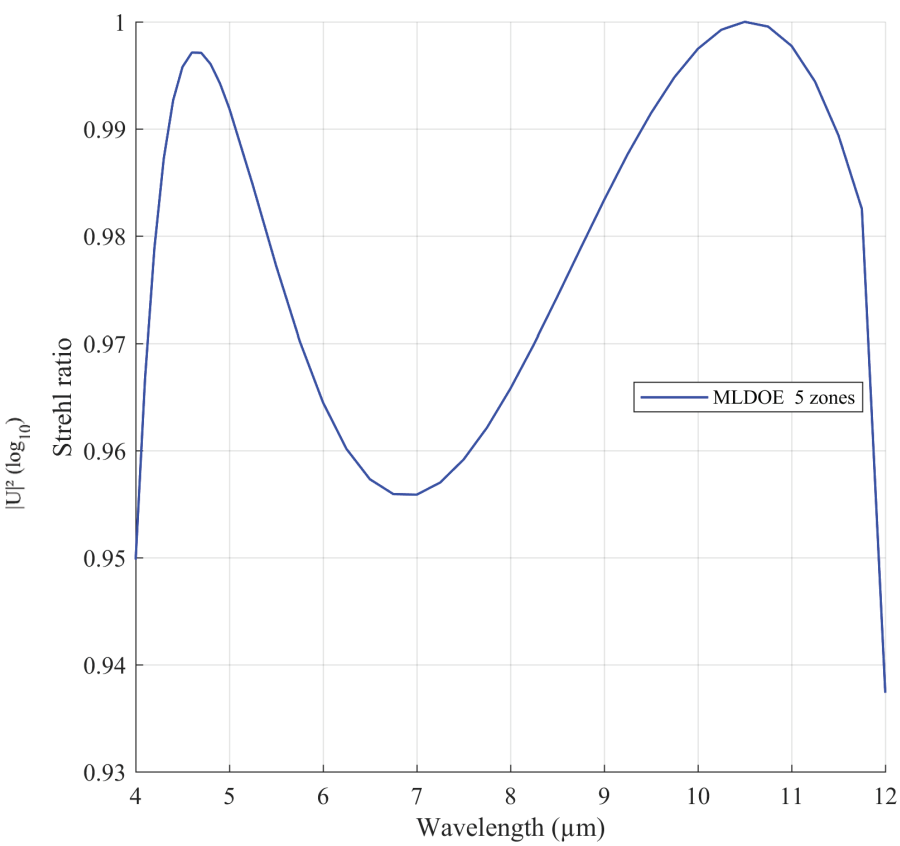

(b) Strehl ratio for the whole bandwidth

Figure 6: Output field in the image plane. The irradiance is displayed with a logarithmic scale. The Strehl ratio is computed at the best focal plane of every wavelength between 4.4 and $12 \mu \mathrm{m}$. 


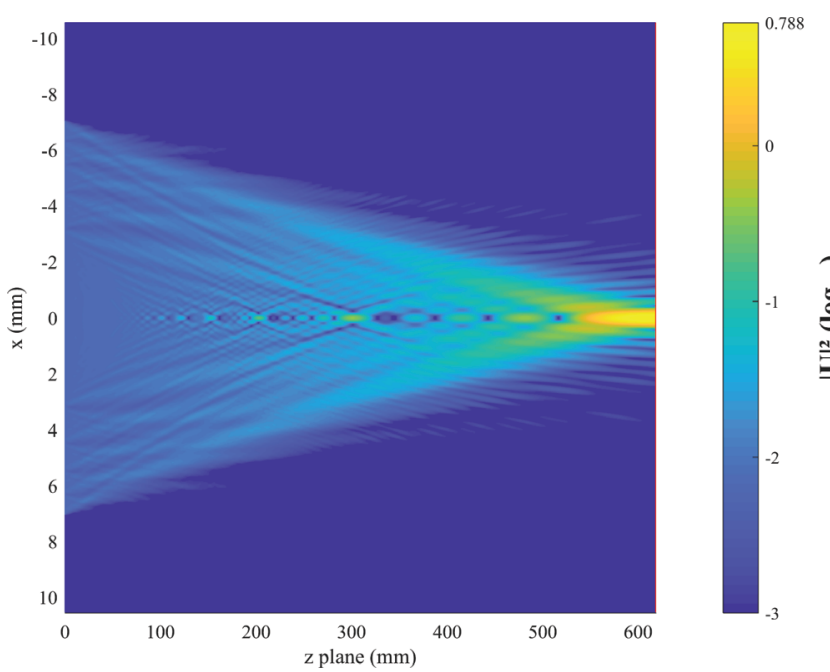

(a) Irradiance $|U(x, y ; z)|^{2}$ along the optical axis at $8 \mu \mathrm{m}$

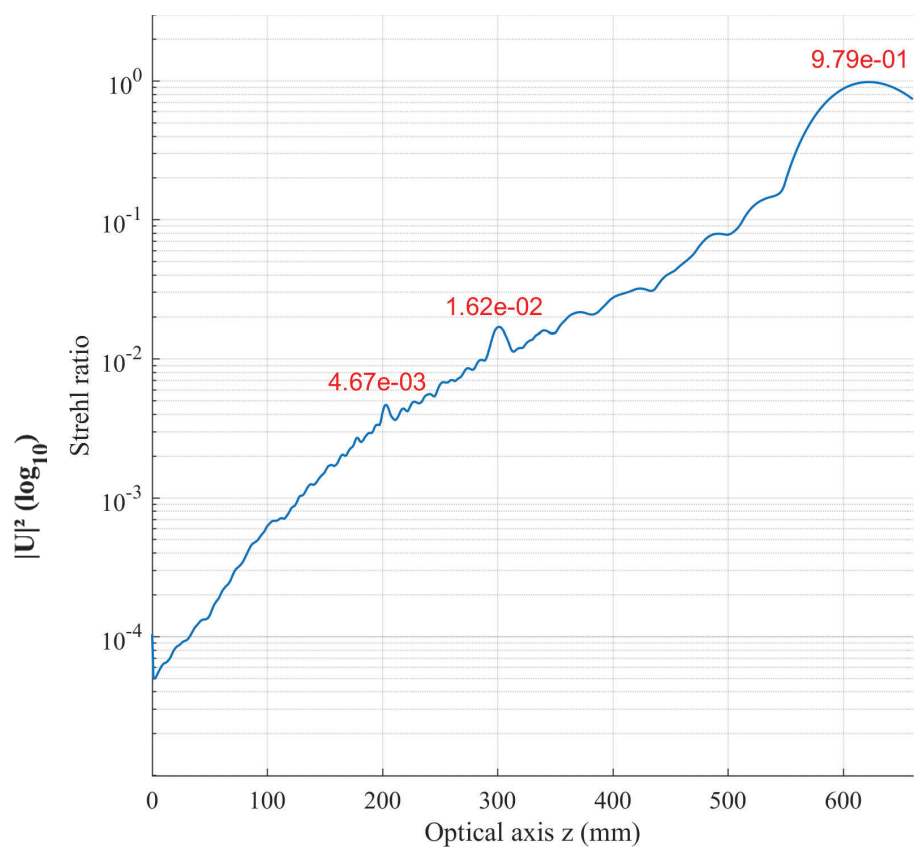

(b) Strehl ratio along the optical axis at $8 \mu \mathrm{m}$ Figure 7: Propagation of the Electric field along the optical axis. The input field resulting from the MLDOE phase shift is situated in the plane $z=0 \mathrm{~mm}$ and the output field is located in the plane $z=620 \mathrm{~mm}$.

As shown in Figure $7 \mathrm{~b}$ ), the peaks have the same location as the diffractive orders $j=+3,+2,+1$, respectively $f, f / 2, f / 3$. These unwanted orders carry nearly no energy. As a consequence, MLDOEs have a unique focus, located at the first diffractive order. This property illustrates the imaging potential of MLDOEs for wide-band applications.

\section{CHROMATIC BEHAVIOUR OF A MLDOE}

In Section 2 the broad-band imaging capabilities of the MLDOE were presented. However, the use of different wavelengths generates a variation of the focal length called "chromatic focal shift". This Section focuses on the MLDOE longitudinal chromatic aberration (LCA). The chromatic compensation of refractive optical elements remains the major use for diffractive optical elements, including MLDOEs. Standard DOE focal length follows a $\frac{1}{\lambda}$ "focal law". Counter-intuitively, in the case of MLDOEs, this relationship holds the same dependency for the wavelength. Thereby, this Section aims to express the focal length $f(\lambda)$ of the MLDOE for any given wavelength $\lambda$.

The two diffractive layers of the MLDOE are HDOEs: they use a higher diffractive order $p_{1}$ and $p_{2}$ respectively. $F_{p_{i}}^{i}$ represents the focal length of the layer $i=1,2$ associated with the order $p_{i}=p_{1}, p_{2}$. Both layers can be considered as thin lenses in contact, therefore the optical power of the MLDOE is the sum of the optical powers of its layers:

$$
\frac{1}{f(\lambda)}=\frac{1}{F_{p_{1}}^{1}(\lambda)}+\frac{1}{F_{p_{2}}^{2}(\lambda)}
$$

The focal law of a HDOE at any diffractive order $j$ and wavelength $\lambda$ is related to its harmonic parameter $p$, aperture radius $R$, number of zones $N$, and design wavelength $\lambda_{0}$ by: ${ }^{18}$

$$
F_{j}(\lambda)=\frac{p \lambda_{0} F_{0}}{j \lambda}=\frac{R^{2}}{2 N j \lambda}
$$


In our case, each HDOE layer as well as the whole MLDOE share the same number of zones and the same aperture radius, so combining Equations 16 and 15 gives:

$$
\frac{1}{f(\lambda)}=\frac{\lambda 2 N}{R^{2}}\left(p_{1}+p_{2}\right)=\left(p_{1}+p_{2}\right) \frac{\lambda}{f_{d} \lambda_{d}}
$$

Where $\lambda_{d}$ is a design wavelength of the MLDOE and $f_{d}$ its associated focal length. Using Equation 17 with $\lambda=\lambda_{d}$ gives: $p_{1}+p_{2}=1$. Therefore, the focal law for a MLDOE is:

$$
f(\lambda)=\frac{f_{d} \lambda_{d}}{\lambda}
$$

This law is numerically verified using Fourier optics: the evaluation of the Strehl ratio along the optical axis (as shown in Figure 7) is performed for multiple wavelengths. For each wavelength, the aim of the propagation is to find the "best focus" plane to derive a law $f_{\text {simu }}(\lambda)$. The simulation domain is confined between the planes $z=f(\lambda)-10 \mathrm{~mm}$ and $z=f(\lambda)+10 \mathrm{~mm}$, where $f(\lambda)$ is defined for each wavelength (from 4.4 to $12 \mu \mathrm{m}$ ) using Equation 18. The "best focus plane" position is determined with an accuracy $\Delta_{z} /(P-1)=32 \mu \mathrm{m}$. Figure 8 shows the comparison between the simulated focal length and the analytical one (Equation 18).

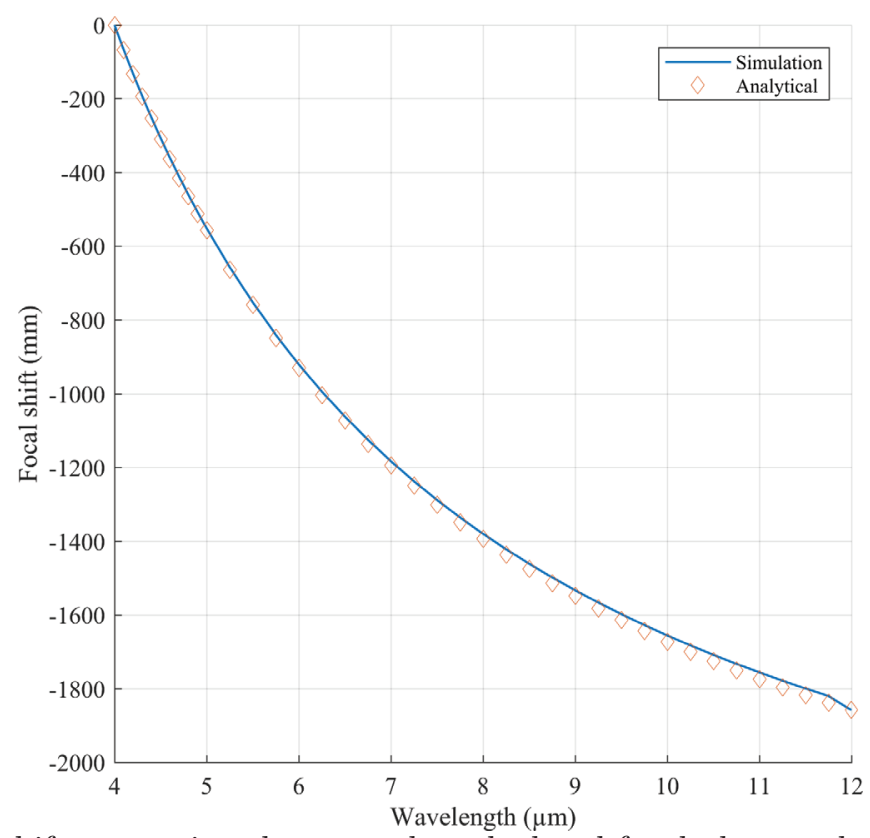

Figure 8: Chromatic focal shift comparison between the calculated focal plane and the determined "best focus" plane. Each point of the simulation curve represents the plane with highest Strehl ratio among 620 planes between $f(\lambda)-10$ and $f(\lambda)+10$ for a given wavelength $\lambda$. The dotted curve shows the expected theoretical behaviour of the MLDOE (Equation 18).

Figure 8 shows a very good agreement between the calculated focal law and the determined best focus. This study shows that, like other DOEs, MLDOEs have a strong negative LCA. Therefore, they combine very well with the refractive components (positive LCA). MLDOEs can replace bulky refractive elements in any broad-band design to correct this aberration.

\section{EFFECTIVE AREA METHOD (EAM)}

In this section, we discuss a recent refined geometric model called effective area method (EAM), which is an application of the extended scalar theory ${ }^{24}$ to the MLDOE. When the aspect ratio of the microstructures becomes high, a non-negligible part of the light is blocked by the vertical edges present in Figure 1 and does not contribute 
anymore to the MLDOE efficiency. This "shadowing effect" is geometrically calculated to refine the expression of the diffraction efficiency, overestimated in the TEA-model. A representation of the EAM model is reported in Figure 9.

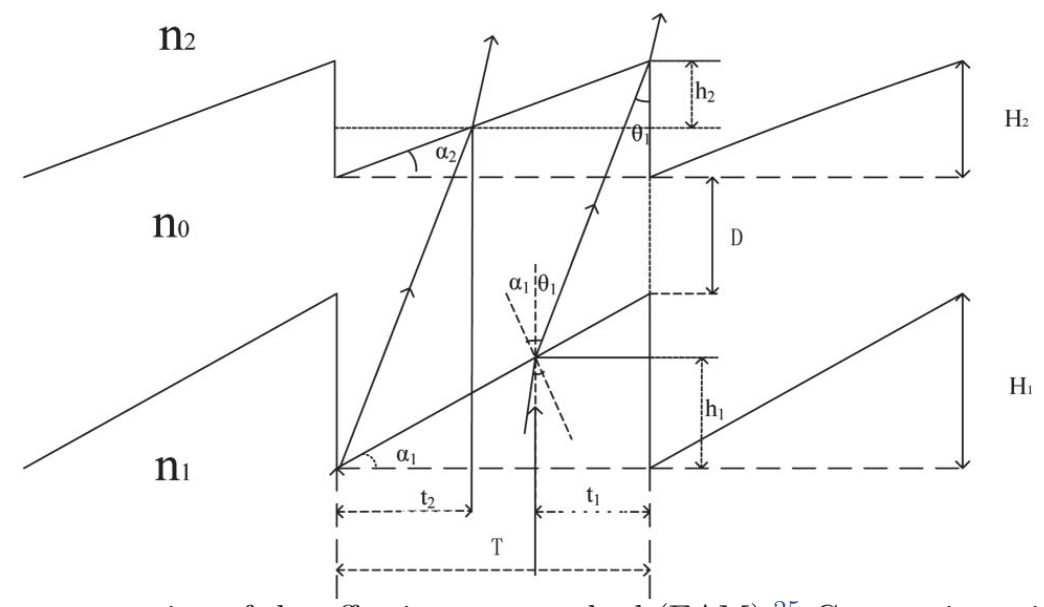

Figure 9: Schematic representation of the effective area method (EAM) ${ }^{25}$ Geometric optic is used to derive the shielded areas $t_{1}$ and $t_{2}$. The optimized microstructure heights $h_{1}$ and $h_{2}$ are now dependant on the period.

This geometric theory gives a diffraction efficiency correction based on the shielded areas $t_{1}, t_{2}$ and the optimized microstructure heights $h_{1}$ and $h_{2}$ :

$$
\eta_{j}(\lambda, T, j)=\operatorname{sinc}^{2}\left(j-\frac{h_{1}\left(n_{1}-1\right)+h_{2}\left(n_{2}-1\right)}{\lambda}\right) * \operatorname{sinc}^{2} t_{1} / T * \operatorname{sinc}^{2} t_{2} / T
$$

The integrated diffraction efficiency over the waveband is often used to compared various models. It is called PIDE (polychromatic integral diffraction efficiency). The PIDE for various period size is plotted in Figure 10 for both EAM and TEA methods :

An average PIDE can be deduced for this MLDOE, integrating the PIDE (EAM) over the period size and dividing by the aperture radius. The resulting average is $83.5 \%$ for the whole MWIR-LWIR wavebands, excepting the atmospheric gap.

\section{CONCLUSION}

In this paper, the need for a dual-band camera is explained for two applications: crop field remote sensing and hot events (forest fires, lava flows) imaging. A solution consisting of two attached diffractive lenses, called multilayer diffractive optical element (MLDOE) is presented. This component is able to keep a very high diffraction efficiency for the whole thermal infrared band $(4.4-12 \mu \mathrm{m})$. Fourier optics is used to provide the image quality of the MLDOE, under the domain of validity of the phase model: the thin element approximation (TEA). The Strehl ratio is very close to 1 for the whole band and the unwanted diffractive orders carry nearly zero energy. Finally, the MLDOE as the same chromatic dispersion as a standard DOE. Therefore, this element is great for achromatization, using a refractive-diffractive combination. MLDOEs have a high potential for broad-band optical design, as an extension of the single-band DOE. Because of the TEA, the studied MLDOE has a very higher $\mathrm{F} / \sharp$ and recent studies such as the effective area method tend to more accurate models, based on geometric optics, to provide a refined estimation of diffraction efficiency and phase delay.

\section{Disclosures}

The results presented in this article have been recently published by the authors in Applied Optics ${ }^{26}$

https://doi.org/10.1364/AO.414082. 


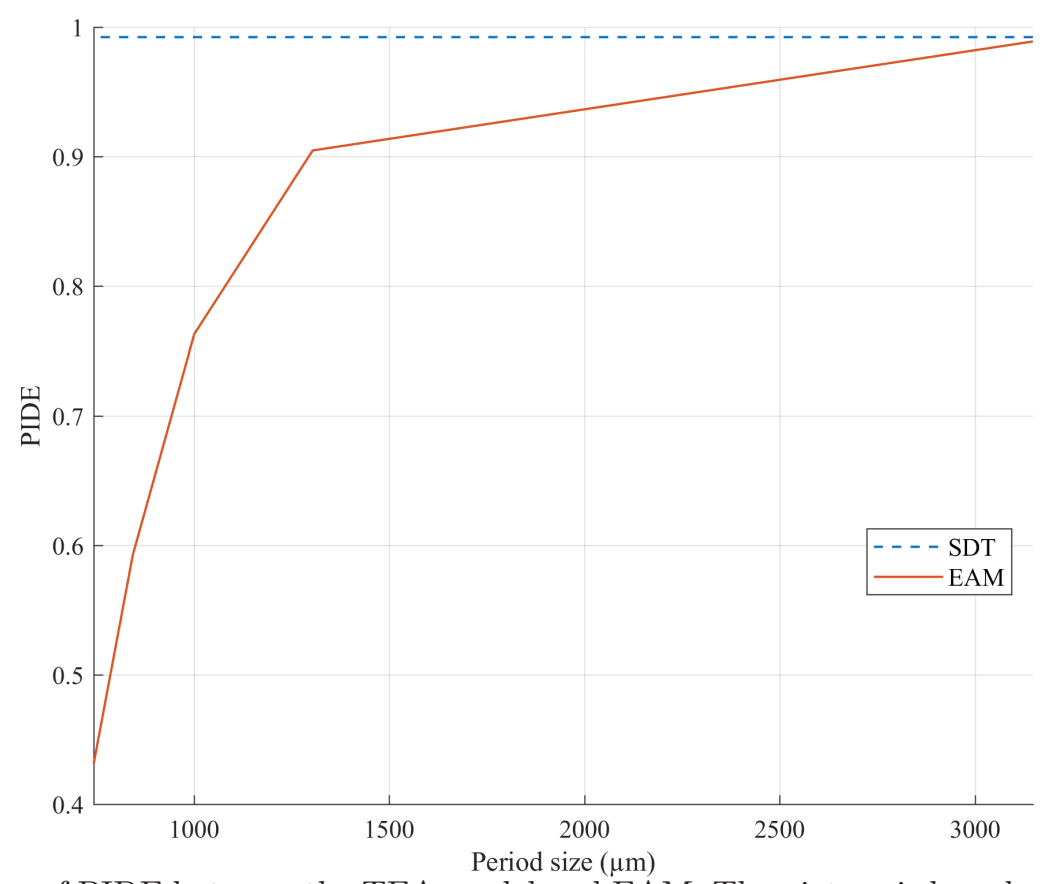

Figure 10: Comparison of PIDE between the TEA model and EAM. The picture is based on the $14 \mathrm{~mm}$ diameter ZnS-Air-Ge MLDOE, with 5 diffractive zones. When the aspect ratio increases (i.e. the period size reduces), the shielded periods $t_{1}$ and $t_{2}$ broaden, explaining the drop of diffraction efficiency.

\section{REFERENCES}

[1] He, X. and Xu, X., "Optimal Band Selection of Multispectral Sensors for Wildfire Detection," in [2017 Sensor Signal Processing for Defence Conference (SSPD)], IEEE (2017).

[2] Spampinato, L., Calvari, S., Oppenheimer, C., and Boschi, E., "Volcano surveillance using infrared cameras," Earth-Science Reviews 106 (2011).

[3] Shimoni, M., "Feasibility study on hyperspectral thermal-infra-red sensor," GSP Research, ESA (2005).

[4] Sobrino, J. A., Del Frate, F., Drusch, M., Jimenez-Munoz, J. C., Manunta, P., and Regan, A., "Review of Thermal Infrared Applications and Requirements for Future High-Resolution Sensors," IEEE Transactions on Geoscience and Remote Sensing 54 (2016).

[5] Tait, H., Rast, M., and Delwart, S., "The optical imaging instruments and their applications: AATSR and MERIS," ESA bulletin 106 (2001).

[6] Pagano, T. S. and Durham, R. M., "Moderate resolution imaging spectroradiometer modis," SPIE 1939 (1993).

[7] Nakajima, T. Y., Nakajima, T., Nakajima, M., Fukushima, H., Kuji, M., Uchiyama, A., and Kishino, M., "Optimization of the Advanced Earth Observing Satellite II Global Imager channels by use of radiative transfer calculations," Applied Optics 37 (1998).

[8] Taghvaeian, S., Chávez, J. L., Altenhofen, J., Trout, T., and DeJonge, K., "Remote sensing for evaluating crop water stress at field scale using infra-red thermography: potential and limitations," Hydrology Days (2013).

[9] Ishimwe, R., Abutaleb, K., and Ahmed, F., "Applications of Thermal Imaging in Agriculture - A Review," Advances in Remote Sensing 03 (2014).

[10] Buralli, D. A., Morris, G. M., and Rogers, J. R., "Optical performance of holographic kinoforms," Applied Optics 28(5) (1989).

[11] Moreno, V., Salgueiro, J. R., and Román, J. F., "High efficiency diffractive lenses: Deduction of kinoform profile," American Journal of Physics (1997). 
[12] Fan, C., Z.Wang, L.Lin, Zhang, M., and Fan, H., "Design of infrared telephoto-optical system with double layer harmonic diffractive element," Chinese Physics Letters 24 (2007).

[13] Xue, C., Cui, Q., Liu, T., Liangliang, Y., and Fei, B., "Optimal design of multilayer diffractive optical element for dual wavebands," Optics Letters 35(24) (2010).

[14] Mao, S., Cui, Q., Piao, M., and Zhao, L., "High diffraction efficiency of three-layer diffractive optics designed for wide temperature range and large incident angle," Applied Optics 55(13) (2016).

[15] Zhang, B., Cui, Q., and Piao, M., "Effect of substrate material selection on polychromatic integral diffraction efficiency for multilayer diffractive optics in oblique incident situation," Optics Communications 415, 156163 (2018).

[16] Takehiko, N. and Hideki, O., "Research on multi-layer diffractive optical elements and their application to camera lenses," DOMO,DMA2 (2002).

[17] Sweeney, C. W. and Sommargren, G. E., "Harmonic diffractive lenses," Applied Optics 34, 2462-2475 (1995).

[18] Faklis, D. and Morris, G. M., "Spectral properties of multiorder diffractive lenses," Applied Optics 34, 2462-2468 (1995).

[19] Goodman, J. W., "The angular spectrum of plane waves," in [Introduction to Fourier Optics], ch. 3, McGraw-Hill (1996).

[20] Matsushima, K. and Shimobaba, T., "Band-limited angular spectrum method for numerical simulation of free-space propagation in far and near fields," Optics Express 12(22) (2009).

[21] Zhong, H., Zhang, S., Wyrowski, F., and Schweitzer, H., "Parabasal thin element approximation for the analysis of the diffractive optical elements," Proc. of SPIE 9131(913114-2) (2014).

[22] Pommet, D. A., Moharam, M., and Grann, E. B., "Limits of scalar diffraction theory for diffractive phase elements," JOSA 11(6) (1994).

[23] Greisukh, G., Danilov, G., Ezhov, E., Stepanov, S., and Usievich, B., "Comparison of electromagnetic and scalar methods for evaluation of efficiency of diffractive lenses for wide spectral bandwidth," Optics Communication 338, 54-57 (2015).

[24] Swanson, G., "Binary optics technology: Theoretical limits on the diffraction efficiency of multilevel diffractive optical elements," tech. rep., Lincoln Laboratory, Massachusetts Institute of Technology (1991).

[25] Yang, H., Xue, C., Li, C., and Wang, J., "Optimal design of multilayer diffractive optical elements with effective area method," Applied Optics 55(7) (2016).

[26] Laborde, V., Loicq, J., and Habraken, S., "Modeling infrared behavior of multilayer diffractive optical elements using fourier optics," Applied Optics 60(6) (2021). 